\title{
Distribution of Heat Stabilizers in Plasticized PVC-Based Biomedical Devices: Temperature and Time Effects
}

\author{
Lidia Maria Bodecchi, ${ }^{1}$ Caterina Durante, ${ }^{2,3}$ Marcello Malagoli, ${ }^{1}$ Matteo Manfredini, ${ }^{1}$ \\ Andrea Marchetti, ${ }^{2,3}$ and Simona Sighinolfi, ${ }^{2,3}$ \\ ${ }^{1}$ Gambro Dasco S.p.A, Via Modenese 30, 41036 Modena, Italy \\ ${ }^{2}$ Department of Chemistry, University of Modena and Reggio Emilia, Via G. Campi 183, 41125 Modena, Italy \\ ${ }^{3}$ INSTM, UdR of Modena, 41125 Modena, Italy \\ Correspondence should be addressed to \\ Andrea Marchetti, andrea.marchetti@unimore.it
}

Received 20 April 2011; Revised 13 June 2011; Accepted 14 June 2011

Academic Editor: Ahmed Aamouche

Copyright (C) 2011 Lidia Maria Bodecchi et al. This is an open access article distributed under the Creative Commons Attribution License, which permits unrestricted use, distribution, and reproduction in any medium, provided the original work is properly cited.

Thermoplastic polymers can be viewed as a dynamic framework in which additives allocation is strongly dependent on the system' chemistry. Considering the complexity of the distribution phenomena that may occur in plastics obtained by blending polymeric resins with different additives, this work constitutes an attempt to the description of the behavior of PVC heat stabilizers (calcium and zinc carboxylates), as regard temperature and time. Thanks to the Fourier Transform Infrared Spectroscopy, it is possible to observe a first decreasing trend of the additives related IR-bands as a function of the increasing temperature and the higher the temperature the faster the decrease of the heat stabilizers intensities bands is, with respect to time. Additives distribution in not sterilized, sterilized, aged not sterilized and aged sterilized materials have been investigated to determine their behavior with respect to temperature, from 30 to $120^{\circ} \mathrm{C}$, and time. A simulated supplementary aging process equivalent to 9 months aging was carried out on aged not sterilized and aged sterilized materials to gain more data on the transport/reaction phenomena these additives in the plastic material. Experimental evidences allow hypothesizing that reaction and redistribution phenomena probably concur to determine the additives allocation in PVC as a function of temperature and time.

\section{Introduction}

Poly(vinyl chloride) (PVC) is one of the most important plastic material available today in terms of global market figures and production volumes, with widespread applications in different areas. The reasons for its worldwide importance, among physical-chemical characteristics, are, on one side, its compatibility with a large number of other products (e.g., plasticizers, heat stabilizers, and lubricants) that are able to modify PVC's chemical and physical properties to obtain from rigid to flexible end products even with complex shapes and, on the other side, the low production costs [1].

The study of different PVC plasticizers has always been a big concern [2-6] for the great amounts of such additives and the potential toxic effects [7-10] which some of them have been charged of in the last decades. On the contrary, less attention has been paid to the investigation of the intrinsic stability of mixture, including PVC resins, plasticizers, and thermal stabilizers, along with their dispersion in the blends, and of all those events which may alter such distribution, that is, additives migration, segregation, or degradation phenomena [11-14]. Among PVC additives, particular attention can be focused to heat or thermal stabilizers. As a matter of fact, it is known that low stability is an inherent property of the polymer that undergoes severe degradation even at relatively low temperatures [15]. In order to prevent the polymer degradation and increase the PVC thermal stability, the use of heat stabilizers is required [1]. The choice of the proper heat stabilizers depends, on one hand, on plasticization, lubrication, and cost parameters and, on the other hand, on the use and applications of the PVC end product $[1,16$, 17]. In particular, for flexible (plasticized) biomedical PVCbased disposables, calcium and zinc carboxylates are the only heat stabilizers permitted by the most important national 
and international ruling bodies, such as the European Pharmacopeia [18].

Far away from being a steady state or showing a fixed structure, thermoplastic polymers can be viewed as a dynamic framework with the long polymeric chains swelled by a system of smaller molecules (those of the plasticizers), wherein other additives, whenever present, are dispersed. The distribution of the additives is strongly dependent on the chemistry of the various components and, as a first approximation, on their relative affinity in terms of apolarpolar or steric hindrance [19]. The bulk-to-surface allocation is also governed by thermodynamics, and it will be expected that a proper amount of energy (thermal, radiative, etc.) could alter or modify such distribution, affecting the final functional properties of the end product. Previous studies [2, 16] highlighted the distribution of additives, such as calcium and zinc carboxylates, as a function of the simulant that comes in contact with different PVC-based blends, from rigid to plasticized ones. At the same time, the photo-oxidation of PVC films, stabilized by calcium and zinc stearates, was studied using gravimetry, IR, and UV-visible spectrometry [20-22]. Moreover, when dealing with zinc and calcium stabilizers, a different and opposite behavior of the additives can be stressed out during the extrusion process; in particular, zinc stearate tends to move, "segregate", towards the metal surface of the die faster than calcium stearate does $[23,24]$.

Thus, heat stabilizers molecules can move within the plasticized resin upon various solicitations coming from the outside environments also at ambient temperature. Therefore, surface properties of materials, and thus the intrinsic distribution of the various additives of the blends, become of utmost relevance when medical devices are concerned $[3,4,12,25]$, and, in particular, when blood contacting items, like blood storage bags, catheters for vascular access, tubing, and so forth, are dealt with. In this case, bearing in mind the large number of processes that a plasticized PVC-based blend has to undergo before a finished medical device is manufactured, a possible segregation or a different distribution of additives from the surface to the bulk of the material or device was supposed, as reported in previous studies $[11,12]$.

In particular, results obtained from different authors [2, $11,12,16,23,24]$ highlighted that the radiation sterilization step seems to induce a different allocation of the metal soaps from the surface to the bulk of the examined tubing components, protecting the PVC resin itself from thermal and ionizing degradation phenomena resulting from the sterilization process. On the other hand, accelerated aging test seems to cause a further thermodynamic-supported segregation of additives from the bulk to the surface of the material on the basis of the different "affinity" of the resin and the heat stabilizers [25]. In fact, surface morphology and additives nature, concentration, and distribution, as well as PVC itself, influence the blood compatibility of the polymer, namely, the haemocompatibility of the blood contact surface/device. Adverse reactions can be triggered by nonhaemocompatible materials when they come in contact with blood such as trombi formation, emboli, and, in general, all the characteristic processes included under the wide field of haemostasis $[3,4,12,26]$. Among the different causes that lie behind the trombogenic characteristics of polymer surfaces, several activation factors and cofactors can be mentioned and in particular the action of many metal ions, such as calcium, zinc, and iron play a fundamental role in blood clotting $[27,28]$. Therefore, the presence of metal soaps additives on the inner surface of tubes in contact with blood is a typical case study, where the monitoring of the processing steps has to account for structural phenomena of the modification and migration of chemical species within a plasticized PVC-blend, whose long-terms effects have not been totally disclosed yet.

The present work constitutes a preliminary attempt and experimental approaches to the description of the possible behavior of heat stabilizers in PVC-based tubing components used for extracorporeal medical disposable devices. In particular, this work is focused on the study of the supposed migration of calcium and zinc soaps in extruded tubes as a function of temperature and time. The analytical technique chosen for this purpose is the Fourier transform infrared spectroscopy (FT-IR) combined with a single reflection accessory for attenuated total reflectance (ATR) golden gate equipped with a heated-diamond top plate. In this configuration, surface-sensitive information may be gained thanks to the combination of the FT-IR with the ATR accessory resulting in a final sampling depth of some micrometers [29, 30]. Moreover, the heated-diamond top-plate, allowed us to study the kinetic distribution of additives as regard to the temperature change. In the actual work, different typologies of products, namely, sterilized, nonsterilized, and aged samples were characterized by FT-IR/ATR measurements.

\section{Experimental Section}

2.1. Materials. The medical device, which the whole study dealt with, is a circuitry for extracorporeal haemodialysis. This disposable allows extracorporeal blood circulation from and to the patient through an artificial kidney via a peristaltic pump during a haemodialysis treatment. Such circuitry is a complex assembly of different components, either molded or extruded.

Samples selected for the present experiments belong to one of the extruded components, that is, the tubing, whose surface exposed to the blood during a dialysis treatment is characterized by one of the largest area among those of the components constituting the assembly. All the selected samples were characterized by the same starting developing formulation and were produced by pilot laboratory equipment. Formulation includes plasticizer (di-(2-ethylhexyladipate), DEHA), thermal stabilizers, such as calcium and zinc carboxylate which present a good miscibility with the used plasticizer, and lubricants and other additives in a very limited amount, below $0.5 \% \mathrm{w} / \mathrm{w}$. The percentage of thermal stabilizers, used for the production of tubing components, is always less than $0.9 \%$ [12]. For the present study, two different typologies of tubing, nonsterilized and sterilized, were selected. Sterilization was performed by means of electron beam irradiation ( $\beta$ irradiation) with a typical exposure dose of $25 \mathrm{kGy}$. From $1 \mathrm{~m}$ tubing sample, sterilized and 
nonsterilized, a tube fragment, $10 \mathrm{~cm}$ long, was dissected, and then two subsections, $1 \mathrm{~cm}$ long, were obtained. Finally, the $1 \mathrm{~cm}$ tube sample was further longitudinally cut into two fragments subjected to FT-IR/ATR analysis. In particular, only the inner surface of each fragment was analyzed, since this is the tubing part that comes in contact with blood during the haemodialysis treatment. follows:

Samples collection and analysis were performed as

(a) within 1 month from tubing production (sterilized and nonsterilized),

(b) after 1 year from tubing production (aged sterilized and aged nonsterilized).

Moreover, one-year aged sterilized and one-year nonsterilized samples, after IR-ATR analysis, were subjected to a simplified protocol for accelerated aging, SPAA, in order to test their behavior with respect to time and temperature. IR spectra were collected by applying the same measuring protocol.

2.2. Simplified Protocol for Accelerated Aging, SPAA. A simplified protocol for accelerated aging (known as "10-degree rule"), based on the Arrhenius' collision theory, has been used as additional aging test [25]. Whit this method a device or material is subjected, for a short time, to stresses that are more severe than normal environmental or operational conditions in order to determine the response of the system under normal-usage conditions over a relatively long time.

The relationship between oven test time, Time $_{T_{1}}$ days,

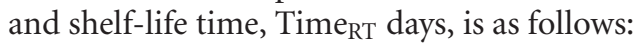

$$
\text { Time }_{T_{1}}=\frac{\text { Time }_{\mathrm{RT}}}{Q_{10} \exp \left(T_{1}-T_{\mathrm{RT}}\right) / 10},
$$

where $T_{1}$ represents the oven aging temperature, ${ }^{\circ} \mathrm{C}, T_{\mathrm{RT}}$ represents the room temperature, ambient or use/storage conditions, ${ }^{\circ} \mathrm{C}$, and $Q_{10}$ is the reaction rate coefficient which is equal to 2 for medical polymers [31, 32].

For the present study, one-year-old samples were stored for 9 days in oven at a temperature of $70^{\circ} \mathrm{C}$ corresponding to an equivalent time of 36 weeks ( 9 months) at normal condition, that is $20^{\circ} \mathrm{C}-22^{\circ} \mathrm{C}$ (ambient temperature). Therefore, after this treatment samples should simulate a 21-month-old tubing.

2.3. Spectroscopic Techniques. Infrared spectra were collected by means of a Bruker VERTEX 70 FT-IR spectrometer equipped with a Mercury Cadmium Telluride, MCT, detector. The instrument was combined with a single reflection ATR accessory (Golden Gate) constituted of a heateddiamond top plate supplied by Specac Ltd. (UK). All the spectra were acquired over 32 scans at a resolution of $1 \mathrm{~cm}^{-1}$ between 4000 and $600 \mathrm{~cm}^{-1}$ and, for each $1 \mathrm{~cm}$ long sampled section; three different measurements were obtained on the inner surface at random locations. Considering the refractive indices of the internal diamond and of a polymer, such as plasticized PVC, to be 2.4 and 1.5 respectively, roughly $2 \mu \mathrm{m}$ depth from the material surface were sampled with an incidence radiation angle of $45^{\circ}$ [29]. Intimate contact between the diamond internal reflectance element and the sample was achieved using a preset torque lock device. FT-IR measurements were carried out at $30^{\circ} \mathrm{C}$, $50^{\circ} \mathrm{C}, 70^{\circ} \mathrm{C}, 90^{\circ} \mathrm{C}$, and $120^{\circ} \mathrm{C}$, respectively, in presence of dry air (dew point $-70^{\circ} \mathrm{C}$ ). Different backgrounds were taken for each temperature, and 3-minute delay time was always hold before starting each measurement. To check the reproducibility of the overall experiments, measurements were always replicated in different days.

\section{Data Analysis}

The spectra collected with the ATR golden gate accessory were normalized by means of a standard normal variate, $\mathrm{SNV}$, procedure, in order to reduce the variability contributions due to the differences in the absolute values of the spectra baselines. The mathematical analyses were performed using Matlab R2006B release, implemented with PLS toolbox 4 [33].

\section{Results and Discussion}

4.1. Temperature Effects. Figure 1 shows the averaged normalized spectra for sterilized (top) and nonsterilized (bottom) samples subjected to FT-IR/ATR measurements carried out at different temperatures, that is, $30^{\circ} \mathrm{C}, 50^{\circ} \mathrm{C}, 70^{\circ} \mathrm{C}, 90^{\circ} \mathrm{C}$, and $120^{\circ} \mathrm{C}$. Focusing on the two different bands related to calcium and zinc carboxylates, $1576 \mathrm{~cm}^{-1}$ and $1540 \mathrm{~cm}^{-1}$, respectively [16], as shown in Figure 2, it is evident that while nonsterilized samples are characterized by the presence of heat stabilizers on the inner surface of the tube at $30^{\circ} \mathrm{C}$, sterilized samples are not. The absence of the bands in the sterilized samples seems to be related to the sterilization process imparted to the tubing component [25]. In fact, high-energy radiation produces scission and crosslinking of the polymer chains, formation of small molecular products, and modification of the chemical structure of the polymer resulting in changes in the materials properties $[11,12$, 32]. In the same way, heat stabilizers are able to prevent the dehydrochlorination reaction of PVC acting as primary and secondary stabilizers [1]. Therefore, the experimental evidence for the sterilized samples seems to confirm the high impact of the $\beta$ sterilization process on the polymer that leads to the heat stabilizers to react its degradation products, such as $\mathrm{HCl}$, at a surface level or by means of their redistribution towards the material in order to prevent the PVC degradation cascade to start or continue.

Figure 2 reports an extended view of the spectral region of interest and shows the effects induced by temperature on the heat stabilizers behavior for nonsterilized samples. In particular, the $1576 \mathrm{~cm}^{-1}$ and $1540 \mathrm{~cm}^{-1}$ bands are present at $30^{\circ} \mathrm{C}, 50^{\circ} \mathrm{C}$, and $70^{\circ} \mathrm{C}$ with a decreasing trend, while at $90^{\circ} \mathrm{C}$ and $120^{\circ} \mathrm{C}$, they disappear. The presence of the calcium/zinc metal soaps on the polymer surface could be mainly due to a kind of chemical incompatibility between the polar PVC backbone and the nonpolar hydrocarbon chain of the stabilizer [25]. As far as the temperature effect 


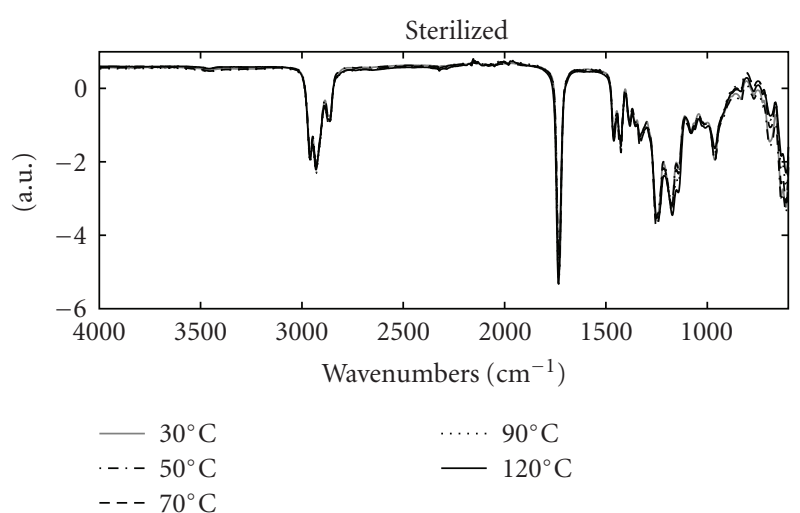

(a)

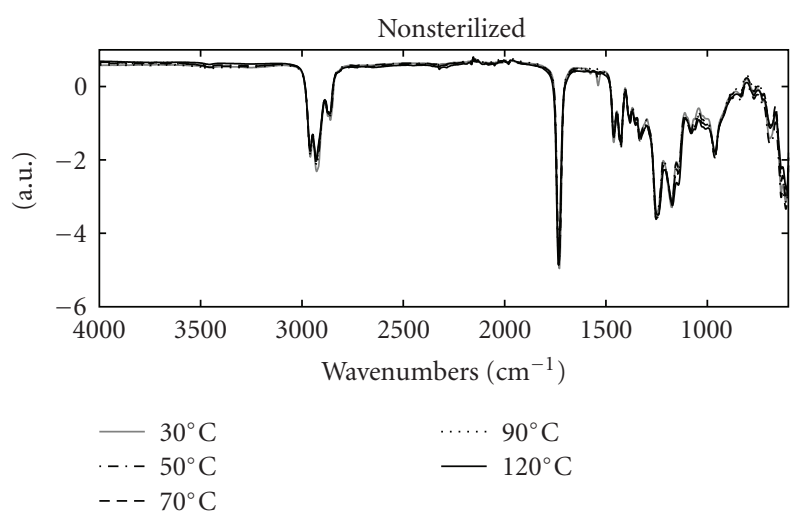

(b)

FIGURE 1: Plot of normalized FT-IR/ATR average spectra, wavelengths $\left(\mathrm{cm}^{-1}\right)$ versus Arbitrary Units (a.u.), (full wave numbers range) of sterilized (a) and nonsterilized samples (b) at 30, 50, 70, 90 and $120^{\circ} \mathrm{C}$, respectively.

is concerned, the decrease of the calcium/zinc carboxylates bands on the inner surface of the tube, both for sterilized and nonsterilized samples, as a function of temperature (Figure 2), could be ascribed to a migration phenomena of the heat stabilizers in the plastic polymer material from the surface to the bulk of the tubes or, otherwise, to reaction mechanisms of the additives themselves as a consequence of the heat treatments. Nevertheless, the latter explanation does not completely justify the experimental evidences obtained for nonsterilized samples at temperatures up to $70^{\circ} \mathrm{C}$, where the $\mathrm{HCl}$ formation should be negligible [34].

\subsection{Temperature Effects: Aged Sterilized and Aged Nonsteril-} ized Samples. The above cited behavior was also observed for nonsterilized samples measured after one year from the tubing production process, aged nonsterilized, as depicted in Figure 3. On the other hand, the appearance of the calcium and zinc soaps related bands on the surface of the aged sterilized tubes at $30^{\circ} \mathrm{C}$ seems to confirm a possible migration phenomena of these species in the plastic polymer probably due to the metal soaps-PVC chemical incompatibility, during the aging process [25].

Figures 4(a) and 4(b) report the comparison of peak areas evaluated for the calcium and zinc stabilizers IR bands

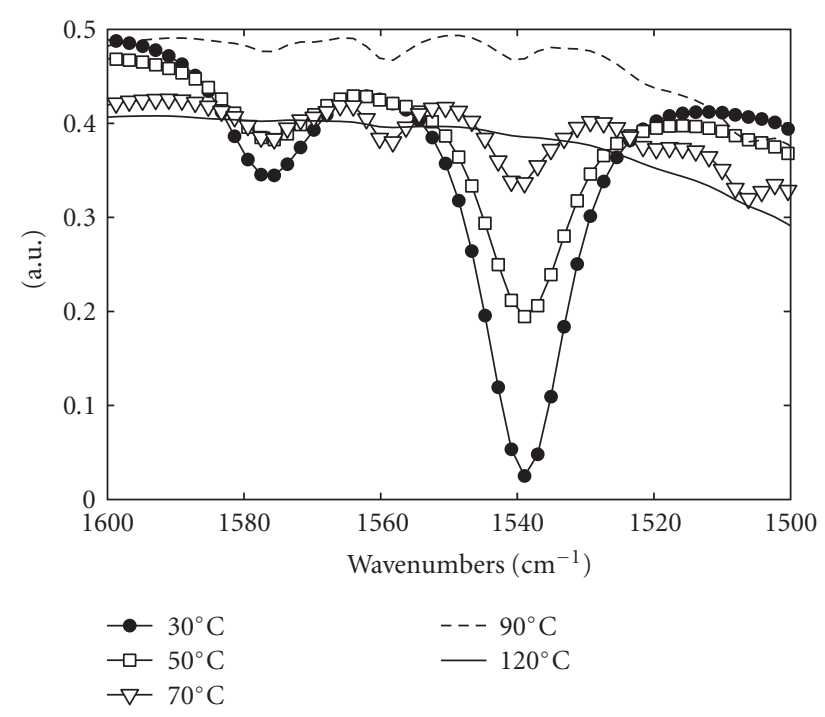

FIGURE 2: Extended view of the normalized FT-IR/ATR average spectra, wavelengths $\left(\mathrm{cm}^{-1}\right)$ versus Arbitrary Units (a.u.), for nonsterilized samples at $30(\bullet), 50(\square), 70(\nabla), 90(--)$, and 120 $(-)^{\circ} \mathrm{C}$, respectively.

for nonsterilized, aged nonsterilized, and aged sterilized samples. Nonsterilized samples present a more intense $1540 \mathrm{~cm}^{-1}$ band, related to the zinc soaps, with respect to $1576 \mathrm{~cm}^{-1}$ band associated to calcium carboxylates. Moreover, calcium soap relative area is larger for aged sterilized samples than for aged nonsterilized ones, while zinc carboxylates relative area is greater for aged nonsterilized samples than for aged sterilized ones. Probably, the different ratio of these additives between aged sterilized and aged nonsterilized samples might be ascribed to the $\beta$ sterilization imparted to the tubing samples. In fact, during the degradation process of PVC, calcium carboxylates, which have little Lewis acidity property, mostly exhibit $\mathrm{HCl}$ scavenger behavior, while zinc soap, which is stronger Lewis acid, not only scavenges $\mathrm{HCl}$, but also substitutes carboxylates for the allylic chlorine atoms [1]. Then, the exchange reaction between zinc chloride and calcium carboxylates regenerates the active zinc additive also avoiding the catalytic effect of zinc chlorides towards PVC degradation [1]. Thus, the reduction of the $\mathrm{Zn}$ soaps on the surface of aged sterilized samples might be correlated to the protection mechanism itself of the heat stabilizers and, consequently, to its initial consumption during the sterilization process. Moreover, when stabilizers are almost totally consumed, polyenes begin to appear, and mass loss takes place [20-22].

In PVC processing equipments, operating at high pressure and temperature, an efficient mixing of all ingredients at molecular level should occur in order to make PVC stable to heat during its processing for short period. Nevertheless, the formation of a thin layer of stabilizers at the surface of the PVC material, that constitutes an interface between the metal surface of the die and the polymer, seems to confirm the migration theory of additives as regard temperature [23, 24]. Thus, migration and thermal stability processes probably concur together to the heat stabilizers distribution in the plastic material as a function of temperature and time. 


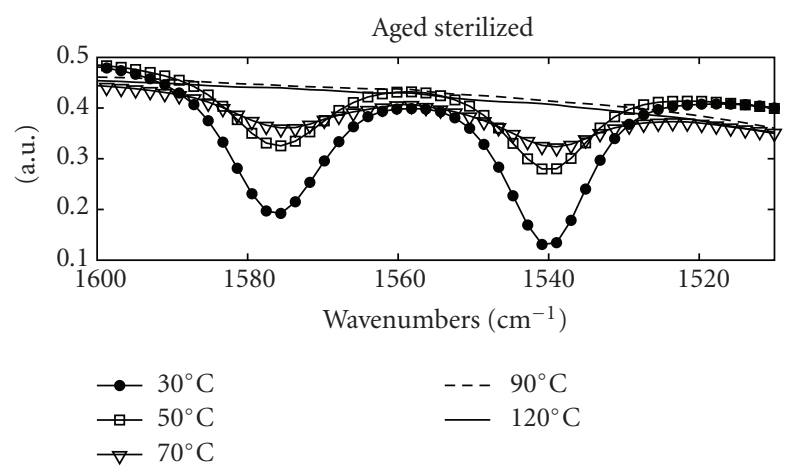

(a)

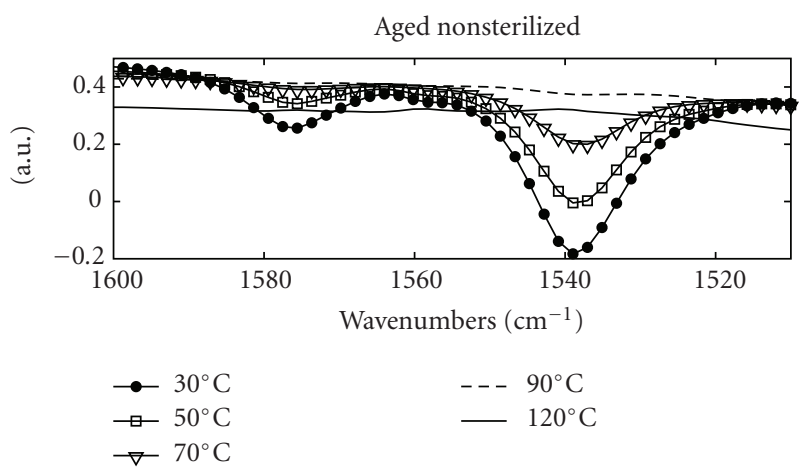

(b)

FIGURE 3: Extended view of the normalized FT-IR/ATR average spectra, wavelengths $\left(\mathrm{cm}^{-1}\right)$ versus Arbitrary Units (a.u.), for one year aged sterilized (a) and one year aged nonsterilized samples (b) at $30^{\circ} \mathrm{C}(\bullet), 50^{\circ} \mathrm{C}(\square), 70^{\circ} \mathrm{C}(\nabla), 90^{\circ} \mathrm{C}(--)$, and $120^{\circ} \mathrm{C}(-){ }^{\circ} \mathrm{C}$, respectively.

4.3. Simplified Protocol Accelerating Aging. To gain more experimental evidences about the additives behavior in the plastic material, aged sterilized, and aged nonsterilized samples, thermally treated during FT-IR/ATR measurements, from $30^{\circ} \mathrm{C}$ to $120^{\circ} \mathrm{C}$, were subsequently subjected to a SPAA process. Figure 5 shows the spectra collected at $30^{\circ} \mathrm{C}$ and $120^{\circ} \mathrm{C}$ for the samples before and after the SPAA process. Spectra collected at $30^{\circ} \mathrm{C}$ for aged samples (not SPAA process applied) present the heat stabilizers related bands that disappear at $120^{\circ} \mathrm{C}$ as previously described. The same samples, after an equivalent aging time of nine months, measured at $30^{\circ} \mathrm{C}\left(\right.$ SPAA $30^{\circ} \mathrm{C}$ ) present the band at $1540 \mathrm{~cm}^{-1}$ correlated to the presence of $\mathrm{Zn}$ carboxylates on the inner surface of the tubes. This behavior could be probably ascribed to a different distribution phenomenon of this additive from the bulk to the surface of the material as a consequence of the supplemental aging process. The fact that only the zinc carboxylates band appeared might confirm the exchange reaction between zinc chloride and calcium carboxylates that regenerates the active zinc carboxylates and, at the same time, consumes the calcium soap that probably reduces its concentration on the surface under the detection limit of the FT-IR/ATR technique. Consumption/exchange reactions and redistribution phenomena of the additives probably occur simultaneously but on a different time scale and, considering

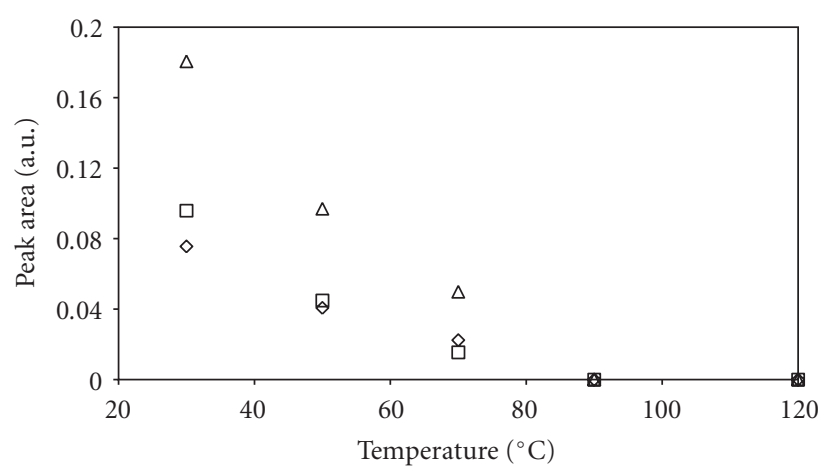

(a)

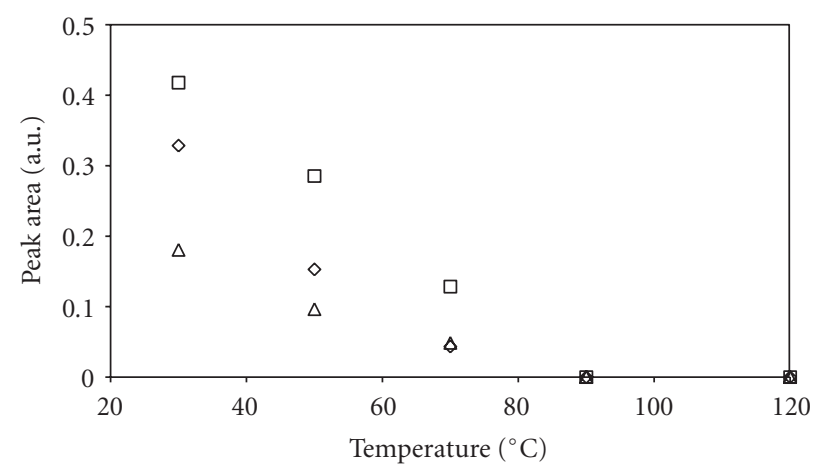

(b)

Figure 4: Plot of the calcium carboxylates $\left(1576 \mathrm{~cm}^{-1}\right)$, (a), and zinc carboxylates $\left(1540 \mathrm{~cm}^{-1}\right)$, (b), peak area at $30^{\circ} \mathrm{C}, 50^{\circ} \mathrm{C}, 70^{\circ} \mathrm{C}$, $90^{\circ} \mathrm{C}$, and $120^{\circ} \mathrm{C}$, respectively, for the nonsterilized $(\diamond)$, one year aged nonsterilized $(\square)$ and one year aged sterilized $(\triangle)$ samples.

the present experimental setup, it is troublesome to show whether the main phenomenon that occurs in the material is the additives reaction and/or their simple migration through the polymer framework.

4.4. Temperature versus Time Effects. Thermal stabilizers behavior was also studied at different temperatures with respect to time, by means of kinetics experiment at different, but constant, temperature. Spectra on sterilized and nonsterilized, aged one year, PVC tubing fragments were collected at different time by heating the samples at $30^{\circ} \mathrm{C}, 50^{\circ} \mathrm{C}$, and $70^{\circ} \mathrm{C}$. Three replicates were acquired for each sample with a time frequency of 10 minutes, and the mean signal of the replicates was considered. Figures 6 and 7 depict the trend of the peaks areas calculated for both the stabilizers IR bands, against the heating time for aged sterilized, Figure 6, and aged nonsterilized, Figure 7, samples, respectively. For the aged sterilized samples at $70^{\circ} \mathrm{C}$, it was possible to follow the thermal stabilizers behavior only for a short time, since the peak area of the IR bands rapidly decrease and the absorption contribution disappears after 10 minutes. Nevertheless, it is possible to observe a decreasing trend, as a function of time, for both samples typologies. Moreover, the higher the temperature, the faster the decrease of the heat stabilizers 


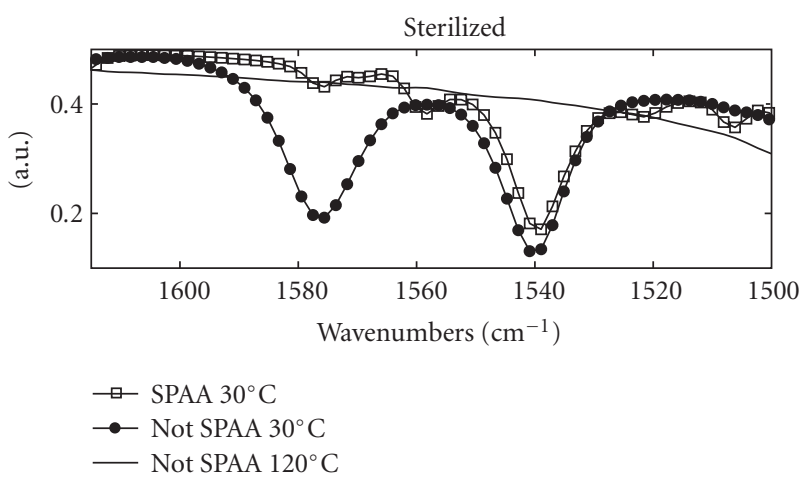

(a)

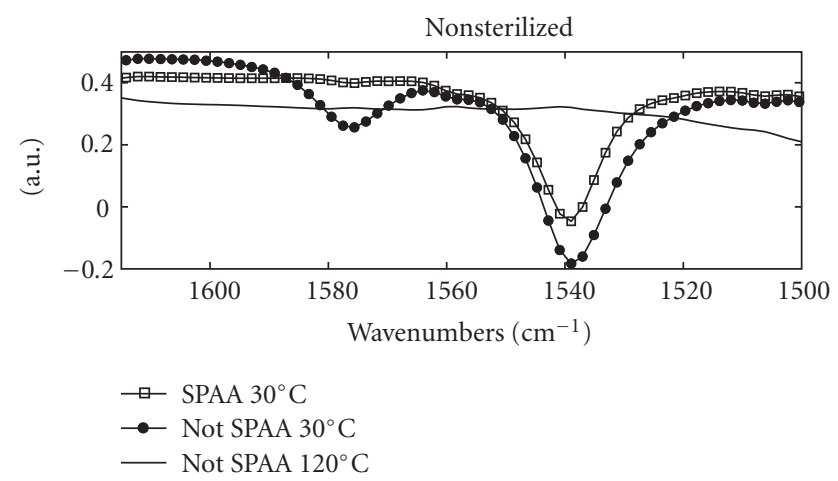

(b)

FIgURE 5: Extended view of the normalized FT-IR/ATR average spectra, wavelengths $\left(\mathrm{cm}^{-1}\right)$ versus arbitrary units (a.u.), of not SPAA and SPAA sterilized samples (a) and not SPAA and SPAA nonsterilized samples (b) at $30^{\circ} \mathrm{C}$ and $120^{\circ} \mathrm{C}$.

bands is. Even in this case, temperature and time play an important role in the distribution phenomena of the calcium and zinc soaps in the plastic material even if PVC degradation processes are not excluded a priori; hence, a reaction between stabilizers degradation products are possible especially at high temperature.

\section{Conclusions}

Aim of this work was the description of the behavior of heat stabilizers in tubing components used for extracorporeal medical disposable devices. In particular, this work was focused on the study of calcium and zinc soaps distribution on the inner surface of extruded tubes as a function of temperature and sample state, namely, nonsterilized/sterilized and aged nonsterilized/sterilized.

In particular, nonsterilized and sterilized materials show a different IR fingerprint when considering the heat stabilizers distribution. The huge decrease of the thermal stabilizers related bands ( 1575 and $1540 \mathrm{~cm}^{-1}$ ) for nonsterilized, aged nonsterilized, and aged sterilized samples from $30^{\circ} \mathrm{C}$ to $120^{\circ} \mathrm{C}$ seems to be correlated to a change in the distribution pattern of the additives from the surface to the bulk of the tube even if increasing the temperature above $70^{\circ} \mathrm{C}$, a

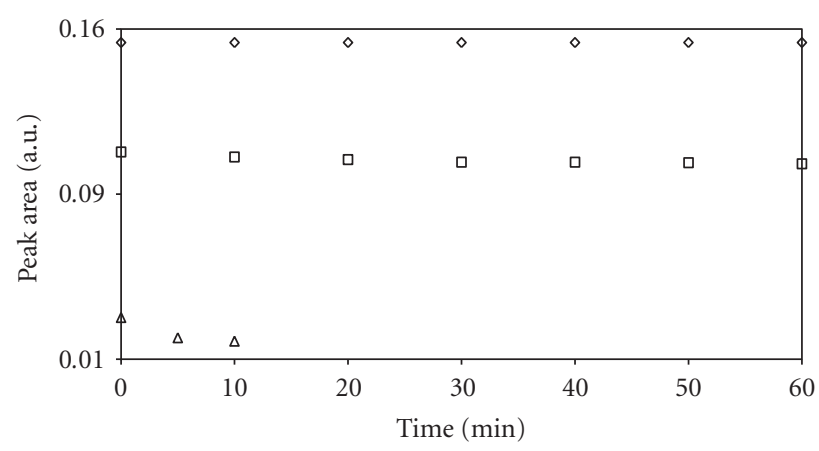

(a)

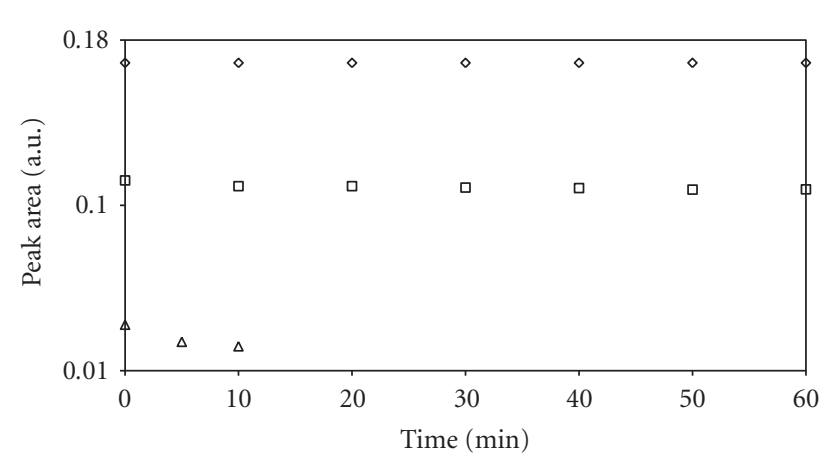

(b)

FIGURE 6: Plot of the calcium carboxylates $\left(1576 \mathrm{~cm}^{-1}\right)$, (a), and zinc carboxylates $\left(1540 \mathrm{~cm}^{-1}\right),(\mathrm{b})$, peak area versus heating time at constant temperature of $30^{\circ} \mathrm{C}(\diamond), 50^{\circ} \mathrm{C}(\square)$, and $70^{\circ} \mathrm{C}(\triangle)^{\circ} \mathrm{C}$ for one year aged sterilized samples.

thermal degradation of PVC-based product probably occurs. Moreover, after a simulated supplementary aging process of 9 months, only the zinc carboxylates band $\left(1540 \mathrm{~cm}^{-1}\right)$ was monitored probably because of the exchange reaction between zinc chloride and calcium carboxylates that regenerates the active zinc carboxylates protecting the resin itself from thermal degradation. The behavior of PVC thermal stabilizers was also studied at different temperatures, $30^{\circ} \mathrm{C}$, $50^{\circ} \mathrm{C}$, and $70^{\circ} \mathrm{C}$, with respect to time. It is possible to observe a decreasing trend of the intensities of calcium and zinc carboxylates bands as a function of time and temperature for both sterilized and nonsterilized aged samples. Results suggest that the behavior of thermal stabilizers is quite complex, and the investigated phenomena, migration, and/or consumption of the additives probably occur simultaneously in the material. In particular, at temperature below $50^{\circ} \mathrm{C}$, it is reasonable to hypothesize that migration phenomena prevail upon reaction mechanisms, since at these temperatures, PVC degradation is quite unexpected. On the contrary, higher temperatures probably induce both consumption and redistribution of the heat stabilizers species within the PVC material. However, further investigations on the role and properties of calcium and zinc soaps in PVC-based formulations for biomedical applications are needed. 


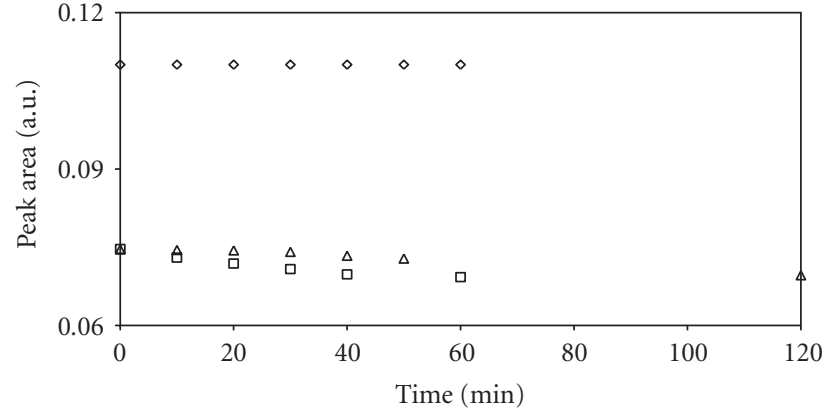

(a)

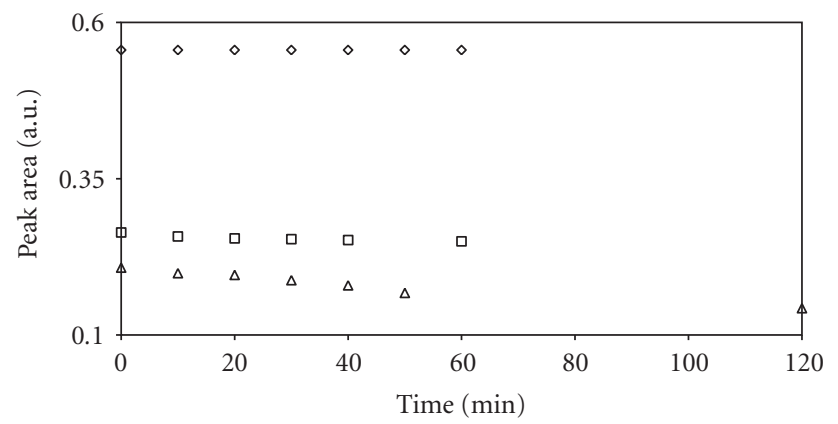

(b)

Figure 7: Plot of the calcium carboxylates $\left(1576 \mathrm{~cm}^{-1}\right)$ (a), and zinc carboxylates $\left(1540 \mathrm{~cm}^{-1}\right)$, (b), peak area versus heating time at constant temperature of $30(\diamond), 50(\square)$ and $70(\triangle){ }^{\circ} \mathrm{C}$ for one year aged nonsterilized samples.

\section{Acknowledgment}

Gambro Dasco S.p.A is kindly acknowledged for the use of its facilities during the production and sampling stages and also for the fruitful collaboration with the University of Modena and Reggio Emilia, Department of Chemistry.

\section{References}

[1] R. Bacalogulu, M. H. Fisch, J. Kaufhold, and H. J. Sander, "PVC Stabilizers," in Plastics Additives Handbook, H. Zweifel, Ed., chapter 3, Munich, Germany, 5th edition, 2001.

[2] M. Hakkarainen, "New PVC materials for medical applications - the release profile of PVC/polycaprolactonepolycarbonate aged in aqueous environments," Polymer Degradation and Stability, vol. 80, no. 3, pp. 451-458, 2003.

[3] R. Ito, F. Seshimo, Y. Haishima et al., "Reducing the migration of di-2-ethylhexyl phthalate from polyvinyl chloride medical devices," International Journal of Pharmaceutics, vol. 303, no. 1-2, pp. 104-112, 2005.

[4] Y. R. Shashoua, "Effect of indoor climate on the rate and degradation mechanism of plasticized poly (vinyl chloride)," Polymer Degradation and Stability, vol. 81, no. 1, pp. 29-36, 2003.

[5] Q. Wang and B. K. Storm, "Separation and analysis of low molecular weight plasticizers in poly(vinyl chloride) tubes," Polymer Testing, vol. 24, no. 3, pp. 290-300, 2005.

[6] Y. B. Liu, W. Q. Liu, and M. H. Hou, "Metal dicarboxylates as thermal stabilizers for PVC," Polymer Degradation and Stability, vol. 92, no. 8, pp. 1565-1571, 2007.
[7] Y. Haishima, R. Matsuda, Y. Hayashi, C. Hasegawa, T. Yagami, and T. Tsuchiya, "Risk assessment of di(2-ethylhexyl)phthalate released from PVC blood circuits during hemodialysis and pump-oxygenation therapy," International Journal of Pharmaceutics, vol. 274, no. 1-2, pp. 119-129, 2004.

[8] K. Kambia, T. Dine, B. Gressier et al., "Evaluation of childhood exposure to di(2-ethylhexyl) phthalate from perfusion kits during long-term parenteral nutrition," International Journal of Pharmaceutics, vol. 262, no. 1-2, pp. 83-91, 2003.

[9] N. M. K. Lamba, J. M. Courtney, J. D. S. Gaylor, and G. D. O. Lowe, "In vitro investigation of the blood response to medical grade PVC and the effect of heparin on the blood response," Biomaterials, vol. 21, no. 1, pp. 89-96, 2000.

[10] X. Zhao and J. M. Courtney, "Influence on blood of plasticized polyvinyl chloride: significance of the plasticizer," Artificial Organs, vol. 23, no. 1, pp. 104-107, 1999.

[11] M. Manfredini, A. Marchetti, D. Atzei et al., "Radiationinduced migration of additives in PVC-based biomedical disposable devices. Part 1. Surface morphology by AFM and SEM/XEDS," Surface and Interface Analysis, vol. 35, no. 4, pp. 395-402, 2003.

[12] M. Manfredini, A. Marchetti, D. Atzei et al., "Radiationinduced migration of additives in PVC-based biomedical disposable devices part 2. Surface analysis by XPS," Surface and Interface Analysis, vol. 35, no. 8, pp. 673-681, 2003.

[13] L.J. Gonzalez-Ortiz, M. Arellano, M.J. Sanchez-Pena, E. Mendizabal, and C.F. Jasso-Gastinel, "Effect of stearate preheating on the thermal stability of plasticized PVC compounds," Polymer Degradation and Stability, vol. 91, no. 11, pp. 27152722, 2006.

[14] D. Briggs, Surface Analysis of Polymer by XPS and Static SIMS, Cambridge University Press, New York, NY, USA, 1998.

[15] W. H. Starnes, "Structural and mechanistic aspects of the thermal degradation of poly(vinyl chloride)," Progress in Polymer Science, vol. 27, no. 10, pp. 2133-2170, 2002.

[16] D. Atek and N. Belhaneche-Bensemra, "FTIR investigation of the specific migration of additives from rigid poly(vinyl chloride)," European Polymer Journal, vol. 41, no. 4, pp. 707714, 2005.

[17] M. Minegawa, "New developments in polymer stabilization," Polymer Degradation and Stability, vol. 25, no. 2-4, pp. 121$141,1989$.

[18] “European pharmacopoeia IV," Section 3.1, 2002.

[19] A. A. A. De Queiroz, É. R. Barrak, and S. C. De Castro, "Thermodynamic analysis of the surface of biomaterials," Journal of Molecular Structure, vol. 394, no. 2-3, pp. 271-279, 1997.

[20] C. Anton-Prinet, G. Mur, M. Gay, L. J. Audouin, and J. Verdu, "Photoageing of rigid PVC-I. Films containing $\mathrm{Ca} / \mathrm{Zn}$ thermal stabiliser," Polymer Degradation and Stability, vol. 60, no. 2-3, pp. 265-273, 1998.

[21] R. Benavides, M. Edge, and N. S. Allen, "The mode of action of metal stearate stabilisers in poly (vinyl chloride): II. Influence of pre-heating on induction times and carbonyl formation," Polymer Degradation and Stability, vol. 49, no. 1, pp. 205-211, 1995.

[22] R. Benavides, M. Edge, and N. S. Allen, "The mode of action of metal stearate stabilisers in poly(vinyl chloride). I. Influence of pre-heating on melt complexation," Polymer Degradation and Stability, vol. 44, no. 3, pp. 375-378, 1994.

[23] I. Fras, P. Cassagnau, and A. Michel, "Lubrication and slip flow during extrusion of plasticized PVC compounds in the presence of lead stabilizer," Polymer, vol. 40, no. 5, pp. 1261$1269,1999$. 
[24] I. Fras, P. Cassagnau, and A. Michel, "Influence of processing conditions on the leaching of thermal stabilizers from plasticized polyvinyl chloride in the presence of water," Journal of Applied Polymer Science, vol. 70, no. 12, pp. 2391-2400, 1998.

[25] L. M. Bodecchi, M. Cocchi, M. Malagoli, M. Manfredini, and A. Marchetti, "Application of infrared spectroscopy and multivariate quality-control methods in PVC manufacturing," Analytica Chimica Acta, vol. 554, no. 1-2, pp. 207-217, 2005.

[26] M. Beltrán, J. C. García, and A. Marcilla, "Infrared spectral changes in PVC and plasticized PVC during gelation and fusion," European Polymer Journal, vol. 33, no. 4, pp. 453-462, 1997.

[27] F. Garbassi, M. Morra, and E. Occhiello, Polymer Surfaces. From physics to Technology, chapter XII, Wiley, Chichester, UK, 1998.

[28] B. D. Ratner, "Biomaterials science: an interdisciplinary endeavor," in Materials in Medicine, B. D. Ratner, A. S. Hoffman, F. J. Schoen, and J. Lemons, Eds., chapter I, Academic Press, San Diego, Calif, USA, 1996.

[29] D. Coombs, "The use of diamond as an ATR material," Vibrational Spectroscopy, vol. 2, pp. 3-5, 1999.

[30] B. Singh and N. Sharma, "Mechanistic implications of plastic degradation," Polymer Degradation and Stability, vol. 93, no. 3, pp. 561-584, 2008.

[31] B. J. Lambert and F. W. Tang, "Rationale for practical medical device accelerated aging programs in AAMI TIR 17," Radiation Physics and Chemistry, vol. 57, no. 3-6, pp. 349-53, 2000.

[32] AAMI, "Compatibility of materials subject to sterilization," Tech. Rep. AAMI TIR17, Association for the Advancement of Medical Instrumentation, Washington Boulevard, Calif, USA, 2008.

[33] C. A. Andersson and R. Bro, "The N-way toolbox for MATLAB," Chemometrics and Intelligent Laboratory Systems, vol. 52 , no. 1, pp. 1-4, 2000.

[34] M. M. Hirschler, "Hydrogen chloride evolution from the heating of poly(vinyl chloride) compounds," Fire and Materials, vol. 29, no. 6, pp. 367-382, 2005. 


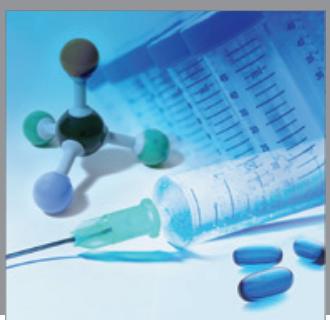

International Journal of

Medicinal Chemistry

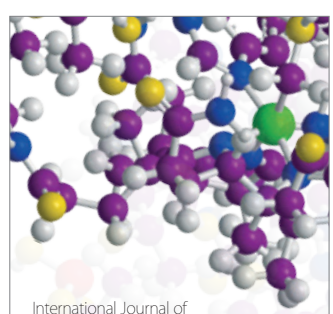

Carbohydrate Chemistry

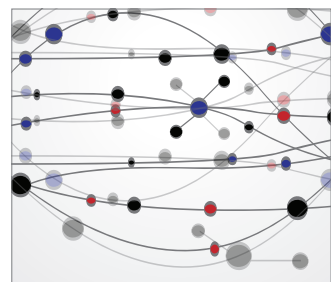

The Scientific World Journal
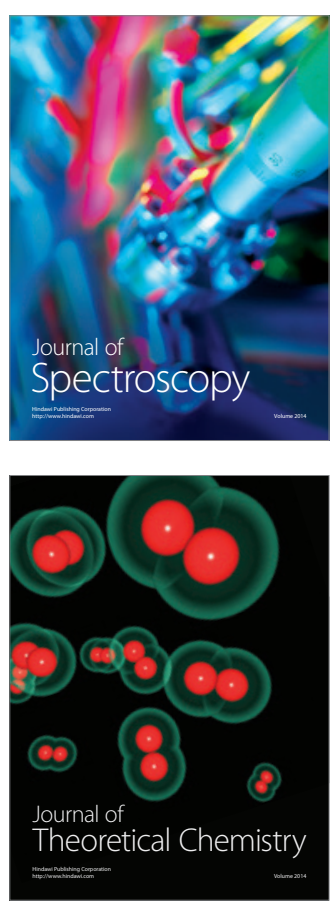
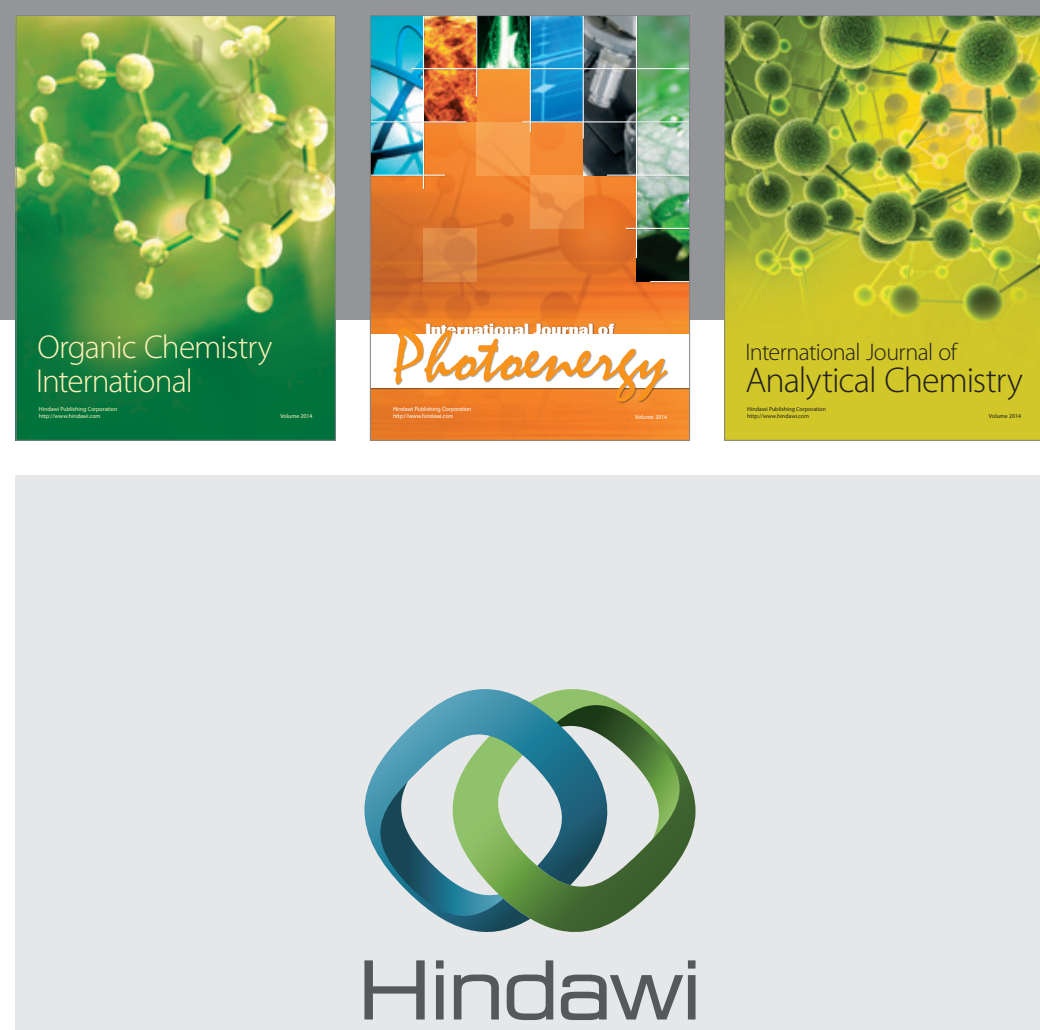

Submit your manuscripts at

http://www.hindawi.com
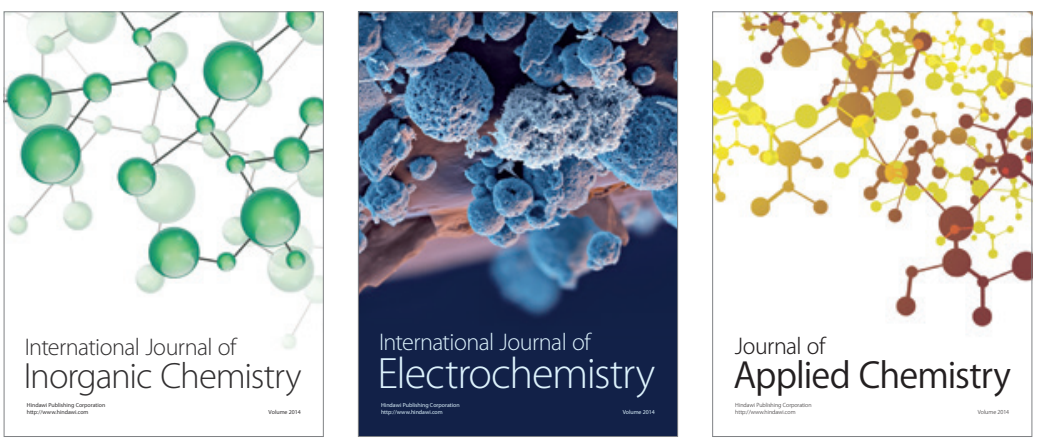

Journal of

Applied Chemistry
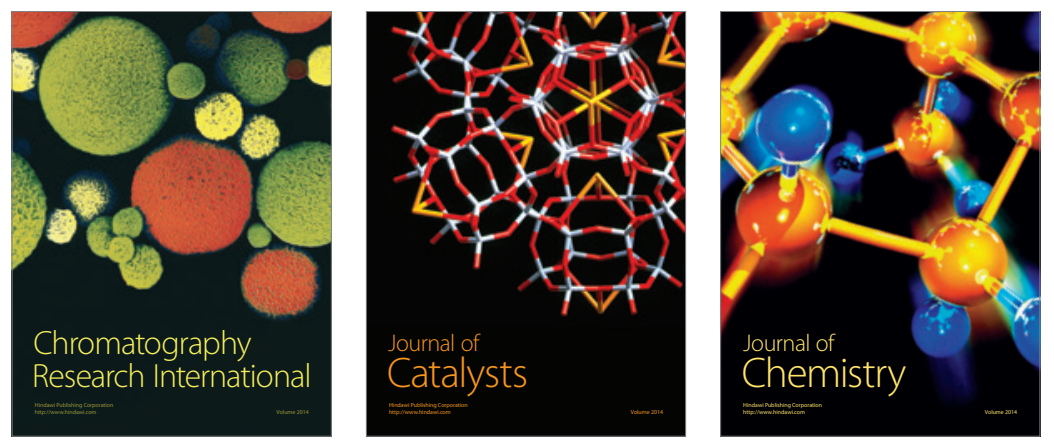
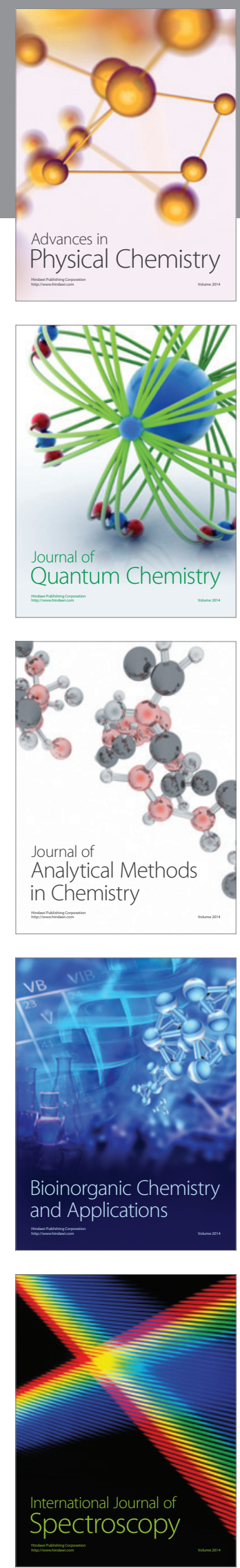